\title{
Mapping the evidence on interventions to raise awareness on lung cancer in resource poor settings: a scoping review protocol
}

\author{
Siyabonga B. Dlamini ${ }^{1 *}$ (D), Benn Sartorius ${ }^{1,2,3}$ and Themba Ginindza ${ }^{1}$
}

\begin{abstract}
Background: Lung cancer is the most commonly diagnosed cancer with $11.6 \%$ of the total cases attributable to lung cancer. It is currently the leading cause of death among cancer-related deaths worldwide. This is a major public health concern. Death due to lung cancer is preventable with interventions encouraging early presentation, diagnosis, smoking cessation and prompt and proper treatment. Literature shows that people are willing to screen for lung cancer if they understand the related risk, because of their behaviour, thus, highlighting the need for tailored interventions to address the associated risks. The aim of the review is to map the available literature on interventions raising community awareness about lung cancer (knowledge, attitudes and health-seeking behaviour) and effectiveness thereof among adults in resource-poor settings.

Methods and analysis: A methodological framework of Arksey and O'Malley will be used to guide this scoping review of published data. This process will start by searching several databases, including the Cochrane Library, PubMed, Scopus, MEDLINE, the Cumulative Index to Nursing and Allied Health Literature (CINAHL), Psyclnfo, Web of Science, Google Scholar and the Educational Resources Information Centre (ERIC). A two-stage process will be done, where, firstly, two reviewers will independently screen the titles and abstracts for eligibility to be included in the final selection of studies. Secondly, a full-text screening of the articles from selected titles and abstracts will then be conducted. A tool developed through an iterative process by the researchers will be used to analyse all bibliographic data and study characteristics of selected studies.
\end{abstract}

Discussion: The results will be used to inform policy and practice in terms of developing interventions on lung cancer awareness. The results of this scoping review will be disseminated through scientific publication, conferences and future workshops with health care professionals involved in lung cancer awareness campaigns.

Keywords: Lung cancer, Community awareness, Interventions, Resource limited settings and effective

\section{Background}

Cancer morbidity and mortality is increasingly becoming a major public health problem, and it is the second leading cause of death worldwide [1]. Globally, over 20 million new cancer cases are projected for 2025 compared to about 14.1 million and 17.5 million new cases in 2012 and 2015, respectively [1-4]. Global cancer cases increased by 33\% between 2005 and 2015 [1]. Lung cancer accounted for about 1.8 million of all cancer cases diagnosed in 2012 [4]. Lung cancer is the most

\footnotetext{
*Correspondence: DlaminiS24@ukzn.ac.za

${ }^{1}$ School of Nursing and Public Health, Discipline of Public Health Medicine,

University of KwaZulu-Natal, Durban, South Africa

Full list of author information is available at the end of the article
}

commonly diagnosed cancer with $11.6 \%$ of the total cases attributable to lung cancer [5]. There were over 1.5 million deaths in 2012 due to lung cancer [4]. Lung cancer is currently the leading cause of death among cancer-related deaths worldwide $[5,6]$ and is thus a major public health concern. Low- and middle-income countries (LMICs) face an increasing challenge of cigarette use and availability of tobacco products [7-9]. Death due to lung cancer is preventable with interventions encouraging early presentation and diagnosis, smoking cessation and prompt and proper treatment [6, 9-11]. Islami and colleagues argued that prevention interventions were needed to curb lung cancer morbidity and mortality in most African

(c) The Author(s). 2019 Open Access This article is distributed under the terms of the Creative Commons Attribution 4.0 International License (http://creativecommons.org/licenses/by/4.0/), which permits unrestricted use, distribution, and 
countries and other LMICs as a result of increased use of tobacco products [9].

A study conducted in Malaysia among smokers and non-smokers showed that people were willing to go for screening, if they were informed that they were particularly at high risk of lung cancer, especially if they were smokers [11]. Whilst prevention is undeniably the best way to address lung and other cancers, challenges brought about by modern lifestyles are not making this approach any easier. For example, modern lifestyles, socio-economic developments and technologically complex human and built environments have profound effects on the scale and profile of cancer; hence, LMICs are now experiencing the greatest increase in cancer incidence [3].

Williams et al. [12] propose multiple interventions including cancer awareness, advocacy, research, workforce care training and funding to avert this situation. Furthermore, there is poor access to quality of care for cancer in SSA, and South Africa is less than optimal [13]. An overwhelming majority of lung cancer patients are diagnosed late and are thus initiated on treatment late. This reduces the effectiveness of treatment, which results in the majority of lung cancer patients dying whilst on treatment [14-17]. This suggests that there are barriers to early diagnosis of lung cancer. These barriers may include (i) structural factors, where patient pathways of care may hinder early diagnosis, (ii) lack of proper health-seeking behaviour, where the patients do not go to health facilities early so that they are screened and initiated on life-saving treatment early, and (iii) cultural beliefs that serve as barriers for individuals to seek help early $[16,18-20]$. Hence, there is a need for better awareness and early detection.

This article outlines the protocol for a scoping review of published literature focusing on interventions that have been proposed and/or implemented to raise community awareness about lung cancer (knowledge, attitudes and health-seeking behaviour) among adults in resource-poor settings.

\section{Methods}

\section{Protocol design}

The scoping review is informed by the framework proposed by Arksey and O'Malley [21] which has been further developed by Levac et al. [22] and the Joanna Briggs Institute [23]. This framework recommends to organise the review process in at least five stages [21]. These stages are (1) identifying the research question, (2) identifying relevant studies, (3) study selection, (4) charting the data, and (5) collating, summarising and reporting the results.

These stages and how they will be applied in the proposed scoping review are discussed below.
Framework stage 1: Identifying the research question The design of this protocol has been informed by the scanning of the existing literature. This exercise assisted in the development of the research question to guide the scope of the review. The broad research question this scoping review will attempt to answer: "What is the effect of community awareness interventions on lung cancer in resource-poor settings?" The review will give an overview of the various interventions available, appraise their effectiveness and identify barriers and facilitators to their effectiveness.

\section{Framework stage 2: Identifying relevant studies}

Identification of studies relevant to this review will be achieved by searching electronic databases of the published literature (including published systematic reviews). These electronic databases include Cochrane $\mathrm{Li}$ brary, PubMed, Scopus, MEDLINE, the Cumulative Index to Nursing and Allied Health Literature (CINAHL), PsycInfo, Web of Science, Google Scholar, EMBASE and the Educational Resources Information Centre (ERIC). All reference lists of included studies will be searched to identify additional relevant studies. A variety of grey literature sources will also be searched to ensure that all relevant information is captured. Relevant grey literature databases (such as Grey Literature Report, OpenGrey, Web of Science Conference Proceedings) will be searched to identify studies, reports and conference abstracts applicable to this review (Additional file 2).

The inclusion criteria will include (i) language of publication: English, (ii) timeframe: no time frame, (iii) study setting: resource-poor setting, and (iv) types of articles: randomised controlled trials, intervention studies (including quasi-experimental designs), reports, literature reviews and evidence maps. Studies will be excluded if they do not meet any of the listed criteria.

Search terms will be determined with input from the research team and in consultation with and academic librarian. However, the following are some of the keywords that are likely to be used to search for articles: lung cancer, awareness, community, interventions, and effective. Terms will be searched as both keywords in the title, abstract and/or subject headings (i.e. $\mathrm{MeSH}$ terms). The review articles retrieved were then screened for their titles, abstracts and index terms. The articles retrieved from each database will be imported into a reference management software, namely EndNote. Endnote will be used for managing records, keeping track of articles and producing a list of references included in the final review report.

\section{Framework stage 3: Study selection}

The review of articles will have two levels of screening. The first level will be title and abstract review, where 
two team members will independently screen the title and abstract of all retrieved citations for inclusion against a set of minimum inclusion criteria; the second, full-text review. Initially, a sample of the retrieved articles will be independently screened by two team members to ensure consistent application of the eligibility criteria for inclusion in the review. Any articles deemed relevant by one or both of the reviewers will be included in the full-text review. Inter-rater reliability will be assessed using percentage agreement. Once the percentage agreement reaches $80 \%$ or above, advancement to the next stage can be done. For lower agreement, the inclusion and exclusion criteria will be clarified. The team will ensure that the duplicates from the different search databases are eliminated. A member of the team will then screen titles and abstracts of the articles to exclude those that do not meet the eligibility criteria identified in the second stage of the protocol. The fulltext articles will be retrieved for the titles and/or abstracts meeting the inclusion criteria.

Disagreements about study eligibility of the sampled articles will be discussed between the two reviewers until a consensus is reached or by arbitration of a third reviewer, if required. The Preferred Reporting Items for Systematic Reviews and Meta-Analyses (PRISMA) flow chart [24-26] will be used to report on the selection process of studies included in this review (see Additional file 1).

\section{Framework stage 4: Charting the data}

Key pieces of information from the abstracts of the selected articles will be collected and sorted. Standard data items will be extracted from the published research literature that will include author, year of publication, study objectives and additional information (i.e. type of study and objectives of the study, intervention strategies on lung cancer awareness and target populations) and will be reported. Based on the preliminary scoping phase, a data extraction framework was developed which will guide the full review articles retrieved from the literature fulfilling the eligibility criteria for inclusion (Table 1). For each article, information on the interventions covered by the review, characteristics of the study populations, setting, length and intensity of the interventions, types of outcomes assessed, information on the effectiveness of the interventions and facilitators and barriers for the implementation of the interventions will be tabled.

The framework will be pilot tested by two team members on a sample (30\%) of the included studies in order to ensure that the coding framework is consistently applied. Additional categories may emerge during the pilot testing process. If necessary, the categories will be modified and the data extraction framework revised accordingly. Questions arising when piloting the framework will be discussed by the team and possible disagreement will be resolved through team consultations. Missing data may be found on some eligible abstracts, and this will be resolved and documented in consultation with the team members. The authors of those articles will be contacted in an attempt to obtain the required details. If there is no response from the authors in 2 to 3 weeks, this will be considered as no information available. However, the study will be included in the narrative with a mention that an attempt was made to contact the authors for the missing data.

Table 1 Data extraction framework

\begin{tabular}{|c|c|c|}
\hline Main category & Sub-category & Description \\
\hline \multicolumn{3}{|l|}{ 1. Authors } \\
\hline \multicolumn{3}{|l|}{ 2. Title } \\
\hline \multicolumn{3}{|l|}{ 3. Journal } \\
\hline \multicolumn{3}{|l|}{ 4. Year of publication } \\
\hline 5. Objectives of the article & & Description of the study objectives \\
\hline 6. Type of study & $\begin{array}{l}\text { Primary (knowledge) } \\
\text { Multiple outcomes (health-seeking } \\
\text { behaviour, knowledge) }\end{array}$ & RCT, quasi-experimental, etc. \\
\hline $\begin{array}{l}\text { 7. Country(ies) where the study } \\
\text { was conducted }\end{array}$ & & Where was this study conducted (Geographical distribution) \\
\hline 8. Language & & Language used in the intervention/publication \\
\hline 9. Target population & $\begin{array}{l}\text { Individual } \\
\text { Community, etc. }\end{array}$ & $\begin{array}{l}\text { Individual or group (community) } \\
\text { Specific age group } \\
\text { Specific sex/gender }\end{array}$ \\
\hline 10. Setting of the intervention & & Community-based, facility-based, urban, rural, resource-poor, etc. \\
\hline 11. Effectiveness & & $\begin{array}{l}\text { Intended intervention outcomes, results presented by authors, future } \\
\text { research directions identified by authors }\end{array}$ \\
\hline
\end{tabular}


The team members that were piloting the framework will be responsible for independently charting the data from each included review study. Similarly, disagreements in extracted data between the two members will be discussed by these members until a consensus is reached or by arbitration of a third reviewer, if required.

\section{Framework stage 5: Collating, summarising and reporting the results}

This review seeks to compile a summary of findings and then propose a "best" model/intervention in a resourcepoor setting broadly present an overview of a particular area of interest. The study will present a narrative account of existing literature using thematic construction based on the different identified intervention types from the selected studies. The analysis of the data collected using the data extraction framework, in this scoping review, will provide information on the body of research that has been conducted on interventions to raise awareness on lung cancer in resource-poor settings. It will be able to provide information on the types of evidence available, the aggregated findings, and offer an outline of the published research rather than an assessment of the quality of individual studies. It would also highlight the effectiveness of the interventions reviewed and their material impact on the target populations. It is also beneficial in identifying gaps in the existing body of knowledge and future research topics.

\section{Discussion}

The results from this scoping review will inform the development of a tailored community lung cancer awareness intervention targeted at selected communities in KwaZulu-Natal, South Africa. This study does not require ethical approval, since the scoping review methodology comprises of reviewing and collecting data from publicly available publications and materials. The results of this scoping review will be disseminated through submitting an article for publication to a scientific journal, conferences, presentations and reports to policymakers and future workshops with professionals involved in lung cancer awareness campaigns. The results will provide a complete synopsis of the interventions on lung cancer awareness and highlight areas where evidence is controversial or missing.

\section{Additional files}

Additional file 1: Proposed PRISMA flowchart. (DOCX 29 kb)

Additional file 2: Draft search strategy and results from three databases. (DOCX $15 \mathrm{~kb}$ )

\section{Abbreviations}

LIMCs: Low- and middle-income countries; MesH: Medical Subject heading; PRISMA: Preferred Reporting Items for Systematic Reviews and MetaAnalyses; RCT: Randomised controlled trial; SSA: Sub-Saharan Africa

\section{Acknowledgements}

Not applicable.

\section{Authors' contributions}

The authors contributed to the development of the research questions, the methods and the drafting and editing. All authors have approved the final manuscript.

\section{Funding}

The lung cancer awareness study was funded by the Bristol-Myres Squibb Foundation (BMSF) under the Secure the Future Initiative.

Availability of data and materials

Not applicable.

Ethics approval and consent to participate

Not applicable.

Consent for publication

Not applicable.

\section{Competing interests}

The authors declared that they have no competing interests.

\section{Author details}

${ }^{1}$ School of Nursing and Public Health, Discipline of Public Health Medicine, University of KwaZulu-Natal, Durban, South Africa. ${ }^{2}$ Health Metrics Sciences, School of Medicine, University of Washington, Seattle, USA. ${ }^{3}$ Faculty of Infectious and Tropical Diseases, Department of Disease Control, London School of Hygiene and Tropical Medicine, London, UK.

Received: 31 January 2019 Accepted: 13 August 2019

Published online: 24 August 2019

\section{References}

1. Global Burden of Disease Cancer C. Global, regional, and national cancer incidence, mortality, years of life lost, years lived with disability, and disability-adjusted life-years for 32 cancer groups, 1990 to 2015: a systematic analysis for the global burden of disease study. JAMA Oncol. 2017;3(4):524-48.

2. Ferlay J, Soerjomataram I, Dikshit R, Eser S, Mathers C, Rebelo M, Parkin DM, Forman D, Bray F. Cancer incidence and mortality worldwide: sources, methods and major patterns in GLOBOCAN 2012. Int J Cancer. 2015;136(5):E359-86.

3. Fidler MM, Soerjomataram I, Bray F. A global view on cancer incidence and national levels of the human development index. Int J Cancer. 2016:139(11):2436-46.

4. Torre LA, Bray F, Siegel RL, Ferlay J, Lortet-Tieulent J, Jemal A. Global cancer statistics, 2012. CA Cancer J Clin. 2015;65(2):87-108.

5. Bray F, Ferlay J, Soerjomataram I, Siegel RL, Torre LA, Jemal A. Global cancer statistics 2018: GLOBOCAN estimates of incidence and mortality worldwide for 36 cancers in 185 countries. CA Cancer J Clin. 2018;68(6):394-424.

6. Schluger NW, Koppaka R. Lung disease in a global context. A call for public health action. Ann Am Thorac Soc. 2014:11(3):407-16.

7. Gilmore AB, Fooks G, Drope J, Bialous SA, Jackson RR. Exposing and addressing tobacco industry conduct in low-income and middle-income countries. Lancet. 2015;385(9972):1029-43.

8. Blecher $\mathrm{E}$, Ross H. Tobacco use in Africa: tobacco control through prevention. Atlanta, GA: American Cancer Society; 2013.

9. Islami F, Torre LA, Jemal A. Global trends of lung cancer mortality and smoking prevalence. Transl Lung Cancer Res. 2015;4(4):327-38.

10. Smith P, Smits S, Owen S, Wood F, McCutchan G, Carter B, Edwards A, Robling M, Townson J, Brain K. Feasibility and acceptability of a cancer symptom awareness intervention for adults living in socioeconomically deprived communities. BMC Public Health. 2018;18(1):695.

11. Loh JF, Tan SL. Lung cancer knowledge and screening in the context of the Malaysian population. JPPR J Pharm Pract Res. 2018:48(1):56-64.

12. Williams CK, Cristina Stefan D, Rawlinson F, Simbiri K, Mbulaiteye SM. The African Organisation for Research and Training in Cancer and its conferences: a historical perspective and highlights of the Ninth 
International Conference, Durban, South Africa, 21-24 November 2013 Ecancermedicalscience. 2014;8:396.

13. Kingham TP, Alatise OI, Vanderpuye V, Casper C, Abantanga FA, Kamara TB, Olopade OI, Habeebu M, Abdulkareem FB, Denny L. Treatment of cancer in sub-Saharan Africa. Lancet Oncol. 2013;14(4):158-67.

14. O'Dowd E, McKeever T, Powell HA, Anwar S, Baldwin D, Hubbard R. Predictors of early death from lung cancer in primary care. Lung Cancer. 2014:83:S34-5.

15. Mack JW, Cronin A, Taback N, Huskamp HA, Keating NL, Malin JL, Earle CC, Weeks JC. End-of-life care discussions among patients with advanced cancer: a cohort study. Ann Intern Med. 2012;156(3):204-10.

16. Birring SS, Peake MD. Symptoms and the early diagnosis of lung cancer. Thorax. 2005;60(4):268.

17. Janssen-Heijnen MLG, van Erning FN, De Ruysscher DK, Coebergh JWW, Groen HJM. Variation in causes of death in patients with non-small cell lung cancer according to stage and time since diagnosis. Ann Oncol. 2015;26(5):902-7.

18. Corner J, Hopkinson J, Fitzsimmons D, Barclay S, Muers M. Is late diagnosis of lung cancer inevitable? Interview study of patients' recollections of symptoms before diagnosis. Thorax. 2005;60(4):314.

19. Corner J, Hopkinson J, Roffe L. Experience of health changes and reasons for delay in seeking care: a UK study of the months prior to the diagnosis of lung cancer. Soc Sci Med. 2006;62(6):1381-91.

20. Wagland R, Brindle L, James E, Moore M, Esqueda Al, Corner J. Facilitating early diagnosis of lung cancer amongst primary care patients: the views of GPs. Eur J Cancer Care. 2017;26:3. https://doi.org/10.1111/ecc.12704.

21. Arksey H, O'Malley L. Scoping studies: towards a methodological framework. Int J Soc Res Methodol. 2005;8(1):19-32

22. Levac D, Colquhoun H, O'Brien KK. Scoping studies: advancing the methodology. Implement Sci. 2010;5:69.

23. Peters M, Godfrey C, Mclnerney P, Soares C, Khalil H, Parker D. The Joanna Briggs Institute reviewers' manual 2015: methodology for JBI scoping reviews; 2015.

24. Liberati A, Altman DG, Tetzlaff J, Mulrow C, Gøtzsche PC, loannidis JPA, Clarke M, Devereaux PJ, Kleijnen J, Moher D. The PRISMA statement for reporting systematic reviews and meta-analyses of studies that evaluate health care interventions: explanation and elaboration. Plos Med. 2009; 6(7):e1000100

25. Moher D, Liberati A, Tetzlaff J, Altman DG, and the PG: Preferred reporting items for systematic reviews and meta-analyses: the PRISMA statement. Annals of Internal Medicine 2009, 151(4):264-269.

26. Moher D, Liberati A, Tetzlaff J, Altman DG. Preferred reporting items for systematic reviews and meta-analyses: the PRISMA statement. Int J Surg. 2010:8(5):336-41.

\section{Publisher's Note}

Springer Nature remains neutral with regard to jurisdictional claims in published maps and institutional affiliations.

Ready to submit your research? Choose BMC and benefit from:

- fast, convenient online submission

- thorough peer review by experienced researchers in your field

- rapid publication on acceptance

- support for research data, including large and complex data types

- gold Open Access which fosters wider collaboration and increased citations

- maximum visibility for your research: over $100 \mathrm{M}$ website views per year

At $\mathrm{BMC}$, research is always in progress.

Learn more biomedcentral.com/submissions 